\title{
Influence of AlN Buffer Layer Deposition Temperature on Properties of GaN HVPE Layers
}

\author{
J. Prażmowska ${ }^{a, *}$, R. Korbutowicz ${ }^{a}$, M. WośKo $^{a}$, R. Paszkiewicz ${ }^{a}$, J. KovaČ $^{b, c}$, \\ R. SRNANEK ${ }^{c}$ AND M. TŁACZAŁA ${ }^{a}$ \\ ${ }^{a}$ Faculty of Microsystem Electronics and Photonics, Wrocław University of Technology \\ Janiszewskiego 11/17, 50-372 Wrocław, Poland \\ ${ }^{b}$ Microelectronics Department, Faculty of Electrical Engineering and Information Technology \\ Slovak University of Technology, Ilkovicova 3, 81219 Bratislava, Slovakia \\ ${ }^{c}$ International Laser Centre, Ilkovicova 3, 81219 Bratislava, Slovakia
}

\begin{abstract}
Gallium nitride layers were deposited on AlN and double layer (AlN/AlGaN) buffers grown at various temperatures on $\mathrm{Al}_{2} \mathrm{O}_{3}$. Stress in layers was evaluated based on the Raman scattering and photoluminescence measurements. The obtained values were less than $1 \mathrm{GPa}$.
\end{abstract}

PACS numbers: 81.10.Bk, 81.15.Gh, 81.15.Kk

\section{Introduction}

Recently gallium nitride appeared to be promising material for application in optoelectronics and electronics. Because of the lack of GaN native crystals alternative substrates are commonly applied. All of them exhibit thermal and lattice mismatch compared to GaN which causes defects generation and strain in deposited layers. Various buffer layers can be applied to minimize the stress which is especially undesirable in optoelectronic devices because it modulates the properties of active layer. The paper presents results of investigation of aluminium buffer layers temperature of deposition influence on the strain in subsequent high temperature GaN layer. Hydride vapor phase epitaxy (HVPE) was applied because it offers the unique possibility to obtain high growth rates and thus, thick layers, which are candidates for free-standing substrates after further delamination.

\section{Experimental details}

High-temperature gallium nitride (HT-GaN) layers were deposited by HVPE on (0001) oriented sapphire substrates with low temperature aluminum nitride (LT-AlN) buffers. The LT-AlNs were deposited at 500, 600, $700{ }^{\circ} \mathrm{C}$ (denoted as sample 500, 600 and 700, respectively) by metalorganic vapor phase epitaxy (MOVPE) technique. Double buffer layer composed of LT-AlN deposited at $600{ }^{\circ} \mathrm{C}$ and followed by $\mathrm{Al}_{x} \mathrm{Ga}_{1-x} \mathrm{~N}$ grown at $960{ }^{\circ} \mathrm{C}$ (denoted as $600 / 960$ ) was also investigated.

* corresponding author; e-mail: Joanna.Prazmowska@pwr.wroc.pl
Before loading into the MOVPE reactor, the substrates were chemically cleaned. Deposition of buffers was preceded by pre-heating of sapphire substrates at $1120^{\circ} \mathrm{C}$ $\left(\mathrm{H}_{2}\right.$ atmosphere, $\left.10 \mathrm{~min}\right)$ and nitridated in $\mathrm{NH}_{3}: \mathrm{H}_{2}$ solution at graded temperature from pre-heating temperature to the temperature of buffer growth. Additionally, aluminium nitride intermediate layer was heated for $10 \mathrm{~min}$ at graded temperature from 600 to $960{ }^{\circ} \mathrm{C}$ in $\mathrm{NH}_{3}: \mathrm{H}_{2}$ solution.

After buffer layers deposition samples were chemically cleaned and heated in HVPE chamber in $\mathrm{NH}_{3}: \mathrm{N}_{2}(1: 10)$ solution for $10 \mathrm{~min}$ at $1050^{\circ} \mathrm{C}$. More details are included in [1]. Temperature of HT-GaN layer growth was $1050^{\circ} \mathrm{C}$ and their thicknesses were to $50 \mu \mathrm{m}$.

The Raman scattering spectra as well as photoluminescence (PL) were measured at room temperature. For the Raman measurements $632.8 \mathrm{~nm}$ line of $\mathrm{He}-\mathrm{Ne}$ laser with beam diameter of about $1 \mu \mathrm{m}$ and its power density of $60 \mathrm{~kW} / \mathrm{cm}^{2}$ was used. Measurements were performed in $x(z, y) \underline{x}$ and $z(x, x) \underline{z}$ geometries where $z$ direction is along $c$-axis and $x$ as well as $y$ perpendicular to the $c$-axis. As a pump beam for PL investigation xenon lamp line of $280 \mathrm{~nm}$ with full width at half maximum (FWHM) of about $10 \mathrm{~nm}$ and the density of excitation power of $1 \mathrm{~mW} / \mathrm{cm}^{2}$, was used. The PL spectra were not normalized to xenon lamp spectra.

\section{Results and discussion}

Morphologies of LT-AlN buffers were similar. Diameter of grains and their heights estimated by atomic force microscopy measurements (not shown) were $10 \mathrm{~nm}$ and $30 \mathrm{~nm}$, respectively. The HT-GaN samples were single 
crystalline as revealed X-ray diffraction measurements (not shown), described earlier [2].

As expected, $\mathrm{E} 2{ }^{\mathrm{H}}$ and $\mathrm{E} 1(\mathrm{TO})$ modes were observed in the Raman scattering spectra in $z(x, x) \underline{z}$ geometry. Absence of A1(LO) modes for almost all samples was possibly caused by high concentration of intrinsic free electrons [3] which could arise from $\mathrm{N}$ vacancies - point defects. This mode occurred only for sample 700, see Fig. 1. Intensities ratios of $\mathrm{E} 2{ }^{\mathrm{H}}$ and $\mathrm{A} 1(\mathrm{LO})$ modes were similar for measurements made in other points (with step of $2 \mu \mathrm{m}$ ) of sample surface (gray and light gray lines) which indicated on uniform concentration of carriers within the layer.

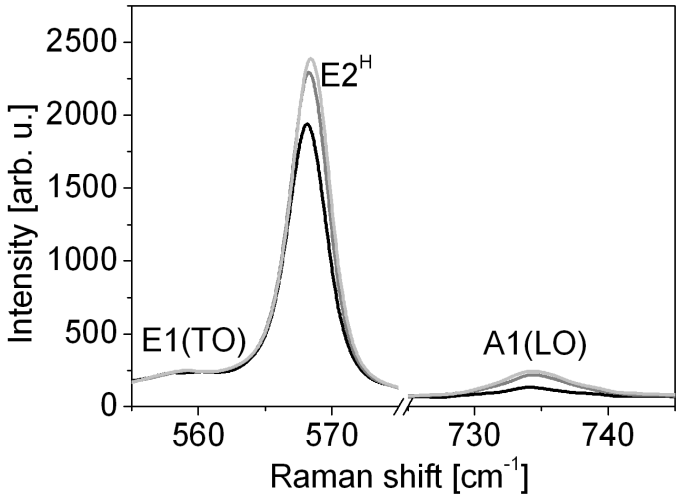

Fig. 1. Raman scattering spectra measured in several points of sample surface (geometry $z(x, x) \underline{z}$ ).

The Raman cross-sectional spectra measured in $x(z, y) \underline{x}$ geometry are depicted in Fig. 2. For all samples $\mathrm{E} 2{ }^{\mathrm{H}}, \mathrm{A} 1(\mathrm{TO})$ and $\mathrm{E} 1(\mathrm{TO})$ modes were observed. The E1(LO) modes expected at about $745 \mathrm{~cm}^{-1}$ were present only for sample 600 (not shown). The aluminium content in double buffer layer was estimated to be below $10 \%$ based on A1(TO) mode shift of sample 600/960 which revealed GaN-like two-mode behaviour.

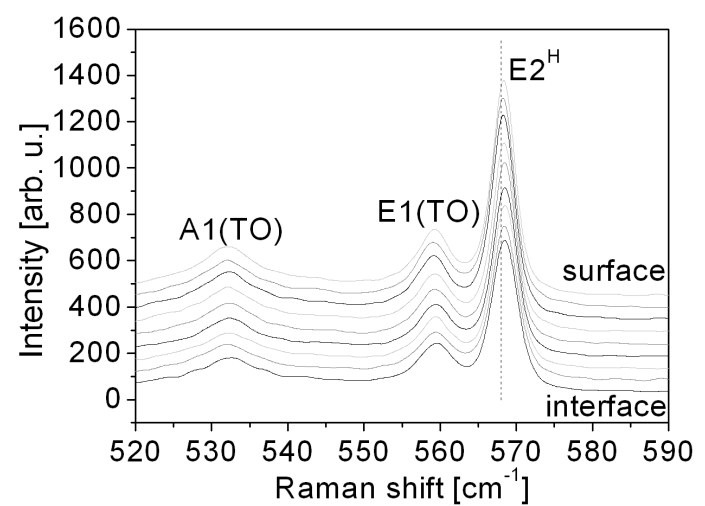

Fig. 2. Raman scattering spectra measured from the substrate to the surface (geometry $z(x, x) \underline{z}$ ).

The FWHM of $\mathrm{E}^{\mathrm{H}}$ mode could be an indicator of the degree of the distortion in a layer [4]. Although $\mathrm{E} 2^{\mathrm{H}}$ mode FWHM is also sensitive to the strain, additionally, the extension of the peak is asymmetrical [3]. Nevertheless values of FWHM (sample $500-2.7 \mathrm{~cm}^{-1}$, sample $600-3.7 \mathrm{~cm}^{-1}$, sample $700-3.33 \mathrm{~cm}^{-1}$, sample $600 / 960-3.83 \mathrm{~cm}^{-1}$ ) confirmed earlier conclusions derived from XRD measurements, described elsewhere [2]. Both samples which consisted of LT-AlN deposited at $600{ }^{\circ} \mathrm{C}$ exhibited poor quality.

Blue shift of $\mathrm{E} 2{ }^{\mathrm{H}}$ mode of $x(z, y) \underline{x}$ spectra indicated on stress occurrence in all layers. Red shift observed in measurements from substrate to the surface arose from the stress vanishing. The shift of mentioned $\mathrm{E} 2{ }^{\mathrm{H}}$ mode as well as PL spectra band-to-band peak enabled biaxial stress evaluation near the surface and interface after Kozawa et al. [5], Fig. 3.

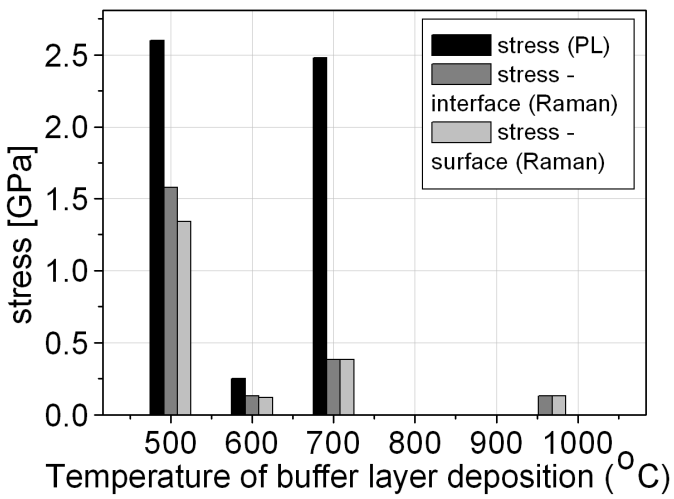

Fig. 3. Stress of gallium nitride layers deposited on template substrates.

Stress values obtained for samples 500 and 700 confirmed conclusions from PL spectra measurements; stress in these samples had the largest value. Comparable values of stress near the surface and interface were observed for samples 600 and 600/960. For calculation, bulk material Raman shift mode was assumed to be $568 \mathrm{~cm}^{-1}$ after Kim et al. [6]. Considering biaxial stress condition, Poisson ratio of $\nu=0.23$ and a Young modulus of $E=290 \mathrm{GPa}$ [7], also $\varepsilon_{\mathrm{c}}$ strain along the $c$-direction was estimated. The values were as follows: $22.8 \times 10^{-4}$ (sample 500$), 1.9 \times 10^{-4}$ (sample 600$), 6.0 \times 10^{-4}$ (sample 700 ), $2.0 \times 10^{-4}$ (sample 600/960). Following formula after Wang et al. [8] theoretical value of strain was calculated to be $21.1 \times 10^{-4}$ which is in good agreement with that occurring in the layer of sample 500 (after assumption of $\Delta \alpha=2.05 \times 10^{-6}{ }^{\circ} \mathrm{C}^{-1}$ and $\Delta T=1030^{\circ} \mathrm{C}$, valid for HVPE technique process conditions). Values of strain corresponded well with that obtained for thick films of GaN presented by Wang et al. [8].

\section{Conclusions}

Gallium nitride layers deposited by HVPE technology on buffered (0001) sapphire substrates with buffers were investigated. As buffer layers LT-AlN films deposited by MOVPE technology at temperatures of $500,600,700^{\circ} \mathrm{C}$ 
and double $\mathrm{AlN} / \mathrm{Al}_{0.1} \mathrm{Ga}_{0.9} \mathrm{~N}$ layers deposited at 600 and $960{ }^{\circ} \mathrm{C}$ were applied. The Raman scattering $\mathrm{E} 2{ }^{\mathrm{H}}$ mode blue shift confirmed occurrence of stress in the layers. The largest value of stress occurred within the layer deposited at $500{ }^{\circ} \mathrm{C}$. Sample deposited at $600{ }^{\circ} \mathrm{C}$ as well as double layer buffer exhibited smaller stress which with connection with revealed earlier poor crystalline quality could lead to the conclusion that those layers were relaxed. Measurements of the Raman shift from the substrate to the surface showed red shift of $\mathrm{E} 2{ }^{\mathrm{H}}$ mode which proved stress vanishing. Additionally absence of A1(LO) mode of the Raman scattering spectra indicated on high concentration of intrinsic carriers in HT-GaN layers, excluding the layer grown on AlN layer deposited at the highest temperature.

\section{Acknowledgments}

This work has been supported in a part by the Polish Ministry of Science and Higher Education under the grants no. R0201802, PBZ-MEiN-6/2/2006, NN 515360436, NN 515053535, by the European Union within European Regional Development Fund, through grant Innovative Economy (POIG.01.01.02-00-008/08) and by Wrocław University of Technology statutory grant.

\section{References}

[1] R. Korbutowicz, E. Dumiszewska, J. Prażmowska, Cryst. Res. Technol. 42, 1297 (2007).

[2] J. Prażmowska, R. Korbutowicz, A. Szyszka, M. Wośko, J. Serafińczuk, R. Paszkiewicz, A. Podhorodecki, J. Misiewicz, J. Kovač, R. Srnanek, M. Tłaczała, J. Phys., Conf. Series 146, 012011 (2009).

[3] M. Klose, R. Dassow, M. Gross, H. Schröder, J. Cryst. Growth 189/190, 666 (1998).

[4] H.P. Ho, K.C. Lo, G.G. Siu, C. Surya, K.F. Li, K.W. Cheah, Mater. Chem. Phys. 81, 99 (2003).

[5] T. Kozawa, T. Kachi, H. Kano, H. Nagase, N. Koide, K. Manabe, J. Appl. Phys. 77, 4389 (1995).

[6] K.S. Kim, C.S. Oh, W.-H. Lee, K.J. Lee, G.M. Yang, C.-H. Hong, E.-K. Suh, K.Y. Lim, H.J. Leee, D.J. Byun, J. Cryst. Growth 210, 505 (2000).

[7] C. Kisielowski, J. Krüger, S. Ruvimov, T. Suski, J.W. Ager III, E. Jones, Z. Liliental-Weber, M. Rubin, E.R. Weber, M.D. Bremser, R.F. Davis, Phys. Rev. B 54, 17745 (1996).

[8] F.C. Wang, C.L. Cheng, Y.F. Chen, C.F. Huang, C.C. Yang, Semicond. Sci. Technol. 22, 896 (2007). 